Algebraic 83 Geometric $\mathcal{T}$ opology

Volume 5 (2005) 1433-1450

Published: 17 October 2005

ATG

\title{
Degree one maps between small 3-manifolds and Heegaard genus
}

\author{
Michel Boileau \\ SHICHENG WANG
}

\begin{abstract}
We prove a rigidity theorem for degree one maps between small 3 -manifolds using Heegaard genus, and provide some applications and connections to Heegaard genus and Dehn surgery problems.
\end{abstract}

AMS Classification $57 \mathrm{M} 50,57 \mathrm{~N} 10$

Keywords Degree one map, small 3-manifold, Heegaard genus

\section{Introduction}

All terminology not defined in this paper is standard, see [He] and Ja].

Let $M$ and $N$ be two closed, connected, orientable 3-manifolds. Let $H$ be a (not necessarily connected) compact 3-submanifold of $N$. We say that a degree one map $f: M \rightarrow N$ is a homeomorphism outside $H$ if $f:(M, M-$ $\left.\operatorname{int} f^{-1}(H), f^{-1}(H)\right) \rightarrow(N, N-\operatorname{int} H, H)$ is a map between the triples such that the restriction $f \mid: M-\operatorname{int} f^{-1}(H) \rightarrow N-\operatorname{int} H$ is a homeomorphism. We say also that $f$ is a pinch and $N$ is obtained from $M$ by pinching $W=f^{-1}(H)$ onto $H$.

Let $H$ be a compact 3-manifold (not necessarily connected). We use $g(H)$ to denote the Heegaard genus of $H$, that is the minimal number of 1-handles used to build $H$.

We define $m g(H)=\max \left\{g\left(H_{i}\right), H_{i}\right.$ runs over components of $\left.H\right\}$. It is clear that $m g(H) \leq g(H)$ and $m g(H)=g(H)$ if $H$ is connected.

A path-connected subset $X$ of a connected 3-manifold is said to carry $\pi_{1} M$ if the inclusion homomorphism $\pi_{1} X \rightarrow \pi_{1} M$ is surjective.

In this paper, any incompressible surface in a 3-manifold is 2-sided and is not the 2-sphere. A closed 3-manifold $M$ is small if it is orientable, irreducible and if it contains no incompressible surface. 
It has been observed by Kneser, Haken and Waldhausen ( $\mathrm{Ha}$, Wa , see also [RW] for a quick transversality argument) that a degree one map $M \rightarrow N$ between two closed, orientable 3-manifolds is homotopic to a map which is a homeomorphism outside a handlebody corresponding to one side of a Heegaard splitting of $N$. This fact is known as "any degree one map between 3-manifolds is homotopic to a pinch".

A main result of this paper is the following rigidity theorem.

Theorem 1 Let $M$ and $N$ be two closed, small 3-manifolds. If there is a degree one map $f: M \rightarrow N$ which is a homeomorphism outside an irreducible submanifold $H \subset N$, then either:

(1) There is a component $U$ of $H$ which carries $\pi_{1} N$ and such that $g(U) \geq$ $g(N)$, or

(2) $M$ and $N$ are homeomorphic.

Remark 1 Given $M$ and $N$ two non-homeomorphic small 3-manifolds, Theorem 1 implies that $N$ cannot be obtained from $M$ by a sequence of pinchings onto submanifolds of genus smaller than $g(N)$. However Theorem 1 does not hold when $M$ is not small. Below are easy examples:

- Let $f: P \# N \rightarrow N$ be a degree one map defined by pinching $P$ to a 3-ball in $N$. Then $f$ is a homeomorphism outside the 3-ball, which is genus zero and does not carry $\pi_{1} N$.

- Let $k$ be a knot in a closed, orientable 3 -manifold $N$ and let $F$ be a once punctured closed surface. Let $M$ be the 3 -manifold obtained by gluing the boundaries of $F \times S^{1}$ and of $E(k)$ in such a way that $\partial F \times\{x\}$ is matched with the meridian of $k, x \in S^{1}$. Then a degree one map $f: M \rightarrow N$ pinching $F \times S^{1}$ to a tubular neigborhood $\mathcal{N}(k)$ of $k$, is a homeomorphism outside a handlebody of genus 1 . If $\pi_{1} N$ is not cyclic or tivial, then $g(\mathcal{N}(k))<g(N)$ and $\mathcal{N}(k)$ does not carry $\pi_{1} N$.

The pinched part of a degree one map between closed, orientable non-homeomorphic surfaces has incompressible boundary [Ed]. The following straigtforward corollary of Theorem 1 gives an analogous result for small 3-manifolds:

Corollary 1 Let $M$ and $N$ be two closed, small, non-homeomorphic 3-manifolds. Let $f: M \rightarrow N$ be a degree one map and let $V \cup H=N$ be a minimal genus Heegaard splitting for $N$. Then the map $f$ can be homotoped to be a homeomorphism outside $H$ such that $f^{-1}(H)$ is $\partial$-irreducible. 
Remark 2 Corollary 1 remains true for any strongly irreducible heegaard splitting of $N$. Then the argument, using Casson-Gordon's result [CG], is essentially the same as [Le, Theorem 3.1], even if in [Le it is only proved for the case $M=S^{3}$ and $N$ a homotopy 3 -sphere. The proof in [Le] is based on his main result [Le, Theorem 1.3], but one can also use a direct argument from degree one maps.

Theorem 1 follows directly from two rather technical Propositions (Proposition (1) and Proposition 21). Theorem [1] and its proof lead to some results about Heegaard genus of small 3-manifolds and Dehn surgery on null-homotopic knots.

Theorem 2 Let $M$ be a closed, small 3-manifold. Let $F \subset M$ be a closed, orientable surface (not necessary connected) which cuts $M$ into finitely many compact, connected 3-manifolds $U_{1}, \ldots, U_{n}$. Then there is a component $U_{i}$ which carries $\pi_{1} M$ and such that $g\left(U_{i}\right) \geq g(M)$.

Remark 3 In general (see La] one has only the upper bound:

$$
\left.g(M) \leq \sum_{i=1}^{n} g\left(U_{i}\right)\right)-g(F) .
$$

Suppose that $k$ is a null-homotopic knot in a closed orientable 3-manifold $M$. Its unknotting number $u(k)$ is defined as the minimal number of self-crossing changes needed to transform it into a trivial knot contained in a 3-ball in $M$.

Theorem 3 Let $k$ be a null-homotopic knot in a closed, small 3-manifold $M$. If $u(k)<g(M)$, then every closed 3-manifolds obtained by a non-trivial Dehn surgery along $k$ is not small. In particular $k$ is determined by its complements.

This article is organized as follows.

In Section 2 we state and prove Proposition 1 which is the first step in the proof of Theorem 1. The second step, given by Proposition 2 is proved in Section 3 , then Theorem 10 follows from these two propositions. Section 4 is devoted to the proof of Theorem 2] and Section [5 to the proof of Theorem 3 .

Acknowledgements We would like to thank both the referee and Professor Scharlemann for their suggestions which enhance the paper. The second author is partially supported by MSTC and NSFC. 


\section{Making the preimage of $H \partial$-irreducible}

The first step of the proof of Theorem 1 is given by the following proposition:

Proposition 1 Let $M$ and $N$ be two closed, connected, orientable, irreducible 3-manifolds which have the same first Betti number, but are not homeomorphic.

Suppose there is a degree one map $f_{0}: M \rightarrow N$ which is a homeomorphism outside a compact irreducible 3 -submanifold $H_{0} \subset N$ with $\partial H_{0} \neq \emptyset$. Then there is a degree one map $f: M \rightarrow N$ which is a homeomorphism outside an irreducible submanifold $H \subset H_{0}$ such that:

- $\partial H \neq \emptyset$;

- $m g(H) \leq m g\left(H_{0}\right)$

- Any connected component of $f^{-1}(H)$ is either $\partial$-irreducible or a 3-ball, and there is at least one component of $f^{-1}(H)$ which is $\partial$-irreducible.

Remark 4 Since $M$ is not homeomorphic to $N$ it is clear that at least one component of $f^{-1}(H)$ is not a 3-ball.

Proof In the whole proof, 3-manifolds $M$ and $N$ are supposed to meet all hypotheses given in the first paragraph of Proposition 1 .

By the assumption there is a degree one map $f_{0}: M \rightarrow N$ which is a homeomorphism outside an irreducible submanifold $H_{0} \subset N$ with $\partial H_{0} \neq \emptyset$.

Let $\mathcal{H}_{0}$ be the set of all 3 -submanifolds $H \subset H_{0}$ such that:

(1) There is a degree one map $f: M \rightarrow N$ which is a homeomorphism outside $H$;

(2) $\partial H \neq \emptyset$;

(3) $m g(H) \leq m g\left(H_{0}\right)$;

(4) $H$ is irreducible.

For an element $H \in \mathcal{H}_{0}$, its complexity is defined as a pair

$$
c(H)=\left(\sigma(\partial H), \pi_{0}(H)\right)
$$

with the lexicographic order, and where $\sigma(\partial H)$ is the sum of the squares of the genera of the components of $\partial H$, and $\pi_{0}(H)$ is the number of components of $H$. 
Remark on $c(H)$ The second term of $c(H)$ is not used in this section, but will be used in the next two sections.

Clearly $\mathcal{H}_{0}$ is not the empty set, since by assumption $H_{0} \in \mathcal{H}_{0}$.

A compressing disk for $\partial H$ in $H$ is a properly embedded 2-disk $(D, \partial D) \subset$ $(H, \partial H)$ such that $\partial D=D \cap \partial H$ is an essential simple closed curve on $\partial H$ (i.e. does not bound a disk on $\partial H$ ). In the following we shall denote by $H \backslash \mathcal{N}(D)$ the compact 3-manifold obtained from $H$ by removing an open product neighborhood of $D$. The operation of removing such neighborhood is called splitting $H$ along $D$.

Lemma 1 Let $H$ be a compact orientable 3-manifold and let $(D, \partial D) \subset$ $(H, \partial H)$ be a compressing disk . Then $m g\left(H_{*}\right) \leq m g(H)$, where $H_{*}=$ $H \backslash \mathcal{N}(D)$ is obtained by splitting $H$ along $D$. Moreover $c\left(H_{*}\right)<c(H)$.

Proof By Haken's lemma for boundary-compressing disk (BO], CG] ), a minimal genus Heegaard surface for $H$ can be isotoped to meet $D$ along a single simple closed curve. It follows that $m g\left(H_{*}\right) \leq m g(H)$.

Since $\partial D$ is an essential simple closed curve on $\partial H$, it is easy to see that $\sigma\left(\partial H_{*}\right)<\sigma(\partial H)$, therefore $c\left(H_{*}\right)<c(H)$.

The proof of Proposition 1 follows from the following:

Lemma 2 Let $H \in \mathcal{H}_{0}$ be an element which realizes the minimal complexity, then any component of $f^{-1}(H)$ which is not a 3 -ball is $\partial$-irreducible.

Proof Let $W_{0} \subset W=f^{-1}(H)$ be a component which is not homeomorphic to a 3 -ball. Such a component exits since $M$ is not homeomorphic to $N$. To prove that $W_{0}$ is $\partial$-irreducible, we argue by contradiction.

If $\partial W_{0}$ is compressible in $W$, there is a compressing disc $(D, \partial D) \rightarrow(W, \partial W)$ whose boundary is an essential simple closed curve on $\partial W$.

Since $f: M \rightarrow N$ is a homeomorphism outside the submanifold $H \subset N$ the restriction $f \mid:(W, \partial W) \rightarrow(H, \partial H)$ maps $\partial W$ homeomorphically onto $\partial H$. Therefore $f(\partial D)$ is an essential simple closed curve on $\partial H$ which bounds the immersed disk $f(D)$ in $H$. By Dehn's Lemma, $f(\partial D)$ bounds an embedded disc $D^{*}$ in $H$.

Lemma 3 By a homotopy of $f$, supported on $W=f^{-1}(H)$ and constant on $\partial W$, we can achieve that: 
- $f \mid: W \rightarrow H$ is a homeomorphism in a collar neighborhood of $\partial W \cup D$,

- $\left.f\right|^{-1}\left(D^{*}\right)=D \cup S$, where $S$ is a closed orientable surface.

Proof We define a homotopy $F: W \times[0,1] \rightarrow H$ by the following steps:

(1) $F(x, 0)=f(x)$ for every $x \in W$;

(2) $F(x, t)=F(x, 0)$ for every $x \in \partial f^{-1}(H)=\partial W$ and for every $t \in[0,1]$;

(3) Then we extend $F(x, 1): D \times\{1\} \rightarrow D^{*}$ by a homeomorphism.

We have defined $F$ on $D \times\{0\} \cup \partial D \times[0,1] \cup D \times\{1\}$ which is homeomorphic to a 2 -sphere $S^{2}$. Since $H$ is irreducible, by the Sphere theorem $\pi_{2}(H)=\{0\}$. Hence:

(4) We can extend $F$ to $D \times[0,1]$;

Now $F$ has been defined on $W \times\{0\} \cup \partial W \times[0,1] \cup D \times[0,1]$, which is a deformation retract of $W \times[0,1]$, therefore:

(5) We can finally extend $F$ on $W \times[0,1]$.

After this homotopy we may assume that $f(x)=F(x, 1)$, for every $x \in W$. Then by construction this new $f$ sends $\partial W \cup D$ homeomorphically to $\partial H \cup D^{*}$. By transversality, we may further assume that $f \mid: W \rightarrow H$ is a homeomorphism in a collar neighborhood of $\partial W \cup D$ and that $\left.f\right|^{-1}\left(D^{*}\right)=D \cup S$, where $S$ is a closed surface.

The following lemma will be useful:

Lemma 4 Suppose $f: M \rightarrow N$ is a degree one map between two closed orientable 3-manifolds with the same first Betti number $\beta_{1}(M)=\beta_{1}(N)$. Then $f_{\star}: H_{2}(M ; \mathbb{Z}) \rightarrow H_{2}(N ; \mathbb{Z})$ is an isomorphism.

Proof Since $f: M \rightarrow N$ is a degree one map, by $[\mathrm{Br}$, Theorem I.2.5], there is a homomorphism $\mu: H_{2}(N ; \mathbb{Z}) \rightarrow H_{2}(M ; \mathbb{Z})$ such that $f_{\star} \circ \mu: H_{2}(N ; \mathbb{Z}) \rightarrow$ $H_{2}(N ; \mathbb{Z})$ is the identity, where $f_{\star}: H_{2}(M ; \mathbb{Z}) \rightarrow H_{2}(N ; \mathbb{Z})$ is the homomorphism induced by $f$.

In particular $f_{\star}: H_{2}(M ; \mathbb{Z}) \rightarrow H_{2}(N ; \mathbb{Z})$ is surjective. Then the injectivity follows from the fact that $H_{2}(M ; \mathbb{Z})$ and $H_{2}(N ; \mathbb{Z})$ are torsion free abelian groups with the same finite $\operatorname{rank} \beta_{2}(M)=\beta_{1}(M)=\beta_{1}(N)=\beta_{2}(M)$. 
Since the degree one map $f: M \rightarrow N$ is a homeomorphism outside $H$, the Mayer-Vietoris sequence and Lemma 4 imply that $f_{\star}: H_{2}(W ; \mathbb{Z}) \rightarrow H_{2}(H ; \mathbb{Z})$ is an isomorphism.

Let $S^{\prime}$ be a connected component of $S$. Since $f\left(S^{\prime}\right) \subset D^{*}$, the homology class $\left[f\left(S^{\prime}\right)\right]=f_{\star}\left(\left[S^{\prime}\right]\right)$ is zero in $H_{2}(H, \mathbb{Z})$. Hence the homology class $\left[S^{\prime}\right]$ is zero in $H_{2}(W, \mathbb{Z})$, because $f_{\star}: H_{2}(W, \mathbb{Z}) \rightarrow H_{2}(H, \mathbb{Z})$ is an isomorphism. It follows that $S^{\prime}$ is the boundary of a compact submanifold of $W$. Therefore $S^{\prime}$ divides $W$ into two parts $W_{1}$ and $W_{2}$ such that $\partial W_{2}=S^{\prime}$ and $W_{1}$ contains $\partial W \cup D$.

We can define a map $g: W \rightarrow H$ such that:

(a) $\left.g\right|_{W_{1}}=\left.f\right|_{W_{1}}$ and $g\left(W_{2}\right) \subset D^{*}$.

Then by slightly pushing the image $g\left(W_{2}\right)$ to the correct side of $D^{*}$, we can improve the map $g: W \rightarrow H$ such that:

(b) $g|\partial W=f| \partial W$,

(c) $g^{-1}\left(D^{*}\right)=D \cup\left(S \backslash S^{\prime}\right)$ and $g: \mathcal{N}(D) \rightarrow \mathcal{N}\left(D^{*}\right)$ is a homeomorphism.

After finitely many such steps we get a map $h: W \rightarrow H$ such that:

(b) $h|\partial W=f| \partial W$,

(d) $h^{-1}\left(D^{*}\right)=D$ and $h: \mathcal{N}(D) \rightarrow \mathcal{N}\left(D^{*}\right)$ is a homeomorphism.

Let $H_{*}=H \backslash \mathcal{N}(D)$ obtained by splitting $H$ along $D$. Then $H_{*}$ is still an irreducible 3 -submanifold of $N$ with $\partial H_{*} \neq \emptyset$.

Now $\left.f\right|_{M-\operatorname{int} W}$ and $\left.h\right|_{W}$ together provide a degree one map $h: M \rightarrow N$. The transformation from $f$ to $h$ is supported in $W$, hence $h$ is a homeomorphism outside the irreducible submanifold $H_{*}$ of $N$.

Since $H_{*}$ is obtained by splitting $H$ along a compressing disk, we have $H_{*} \subset H_{0}$ and $H_{*}$ belongs to $\mathcal{H}_{0}$. Moreover $m g\left(H_{*}\right) \leq m g(H)$ and $c\left(H_{*}\right)<c(H)$ by Lemma 1 .

This contradiction finishes the proof of Lemma 2 and thus the proof of Proposition 1.

\section{Finding a closed incompressible surface in the do- main}

Since closed, orientable, small 3-manifolds are irreducible and have first Betti number equal to zero, Theorem 1 is a direct corollary of the following proposition: 
Proposition 2 Let $M$ and $N$ be two closed, connected, orientable, irreducible 3-manifolds whith the same first Betti number. Suppose that there is a degree one map $f: M \rightarrow N$ which is a homeomorphism outside an irreducible submanifold $H_{0} \subset N$ such that for each connected component $U$ of $H_{0}$, either $g(U)<g(N)$ or $U$ does not carry $\pi_{1} N$. Then either $M$ contains an incompressible orientable surface or $M$ is homeomorphic to $N$.

Let $(M, N)$ be a pair of closed orientable 3-manifolds such that there is a degree one map from $M$ to $N$. We say that condition $(*)$ holds for the pair $(M, N)$ if:

$$
\text { (*) } \quad \pi_{1} N=\{1\} \text { implies } M=S^{3} .
$$

For the proof we first assume that condition $(*)$ holds for the pair $(M, N)$.

\section{Proof of Proposition 2 under condition $(*)$}

By the assumptions, there is a degree one map $f: M \rightarrow N$ which is a homeomorphism outside an irreducible submanifold $H_{0} \subset N$ with $\partial H_{0} \neq \emptyset$ and such that for each connected component $U$ of $H_{0}$ either $g(U)<g(N)$ or $U$ does not carry $\pi_{1} N$. We assume moreover that $M$ is not homeomorphic to $N$. Our goal is to show that $M$ contains an incompressible surface.

Similar to Section 2, let $\mathcal{H}$ be the set of all 3-submanifolds $H \subset N$ such that:

(1) There is a degree one map $f: M \rightarrow N$ which is a homeomorphism outside $H$.

(2) $\partial H$ is not empty.

(3) For each component $U$ of $H$, either $g(U)<g(N)$ or $U$ does not carry $\pi_{1} N$.

(4) $H$ is irreducible.

The set $\mathcal{H}$ is not empty by our assumptions.

The complexity $c(H)=\left(\sigma(\partial H), \pi_{0}(H)\right)$ for the elements of $\mathcal{H}$ is defined like in Section 2 .

Lemma 5 Assume that there is a degree one map $f: M \rightarrow N$ which is a homeomorphism outside a submanifold $H \subset N$. If $H$ contains 3-ball component $B^{3}$, then $f$ can be homotoped to be a homeomorphism outside $H_{*}$, where $H_{*}=H-B^{3}$. Moreover if $H$ is irreducible, then $H_{*}$ is also irreducible. 
Proof By our assumption, there is a degree one map $f: M \rightarrow N$ which is a homeomorphism outside a submanifold $H \subset N$ and $H$ contains a $B^{3}$ component. Since $f \mid: f^{-1}(\partial H) \rightarrow \partial H$ is a homeomorphism, then $f^{-1}\left(\partial B^{3}\right)$ is a 2-sphere $S_{*}^{2} \subset M$. Since $M$ is irreducible, $S_{*}^{2}$ bounds a 3 -ball $B_{*}^{3}$ in $M$. Then either

(a) $M-\operatorname{int} f^{-1}\left(B^{3}\right)=B_{*}^{3}$, or

(b) $f^{-1}\left(B^{3}\right)=B_{*}^{3}$.

In case (a), $N=f\left(B_{*}^{3}\right) \cup B^{3}$ is a union of two homotopy 3-balls with their boundaries identified homeomorphically, and clearly $\pi_{1} N=\{1\}$. So $M=S^{3}$ by assumption $(*)$. Hence (b) holds in either case.

In case (b), by a homotopy of $f$ supported in $f^{-1}\left(B^{3}\right)$, we can achieve that $f \mid: f^{-1}\left(B^{3}\right) \rightarrow B^{3}$ is a homeomorphism. Then $f$ becomes a homeomorphism outside the irreducible 3 -submanifold $H_{*} \subset N$, obtained from $H$ by deleting the 3-ball $B^{3}$.

The last sentence in Lemma 5 is obviously true.

Let $H \in \mathcal{H}$ be an element which realizes the minimal complexity. By Lemma 5 no component of $H$ is a 3 -ball, hence no component of $\partial H$ is a 2 -sphere since $H$ is irreducible. Therefore no component of $f^{-1}(H)$ is a 3-ball and $\partial f^{-1}(H)$ is incompressible in $f^{-1}(H)$ by the proof of Lemma 2

Since $f: M-\operatorname{int} f^{-1}(H) \rightarrow N-\operatorname{int} H$ is a homeomorphism, $\partial f^{-1}(H)$ is incompressible in $M-\operatorname{int} f^{-1}(H)$ if and only if $\partial H$ is incompressible in $N-$ $\operatorname{int} H$. For simplicity we will set $V=N-\operatorname{int} H$, then $N=V \cup H$.

Then the proof of Proposition 2 under condition $(*)$ follows from:

Lemma 6 If $\partial H$ is compressible in $V$, then there is $H_{*} \in \mathcal{H}$ such that $c\left(H_{*}\right)<c(H)$.

Proof Suppose $\partial H$ is compressible in $V$. Let $(D, \partial D) \subset(V, \partial V)$ be a compressing disc. By surgery along $D$, we get two submanifolds $H_{1}$ and $V_{1}$ as follows:

$$
H_{1}=H \cup \mathcal{N}(D), \quad V_{1}=V \backslash \mathcal{N}(D) .
$$

Since $H_{1}$ is obtained from $H$ by adding a 2-handle, for each component $U^{\prime}$ of $H_{1}$ there is a component $U$ of $H_{0}$ such that $g\left(U^{\prime}\right) \leq g(U)$ and $\pi_{1} U^{\prime}$ is a quotient of $\pi_{1} U$, hence $H_{1}$ verifies the defining condition (3) of $\mathcal{H}$. Moreover $f$ is still a homeomorphism outside $H_{1}$ because $H_{1}$ contains $H$ as a subset. 
Clearly $\partial H_{1} \neq \emptyset$. Hence $H_{1}$ satisfies also the defining conditions (1) and (2) of $\mathcal{H}$. We notice that $c\left(H_{1}\right)<c(H)$ because $\sigma\left(\partial H_{1}\right)<\sigma(\partial H)$.

We will modify $H_{1}$ to become $H_{*} \in \mathcal{H}$ with $c\left(H_{*}\right) \leq c\left(H_{1}\right)$. The modification will be divided into two steps carried by Lemma 8 and Lemma 9 below. First the following standard lemma will be useful:

Lemma 7 Suppose $U$ is a connected 3-submanifold in $N$ and let $B^{3} \subset N$ be a 3-ball with $\partial B^{3}=S^{2}$.

(i) Suppose $S^{2} \subset \partial U$. If int $U \cap B^{3} \neq \emptyset$, then $U \subset B^{3}$. Otherwise $U \cap B^{3}=S^{2}$.

(ii) if $\partial U \subset B^{3}$, then either $U \subset B^{3}$, or $N-\operatorname{int} U \subset B^{3}$.

Proof For (i): Suppose first $\operatorname{int} U \cap B^{3} \neq \emptyset$. Let $x \in \operatorname{int} U \cap B^{3}$. Since $U$ is connected, then for any $y \in U$, there is a path $\alpha \subset U$ connecting $x$ and $y$. Since $S^{2}$ is a component of $\partial U, \alpha$ does not cross $S^{2}$. Hence $\alpha \subset B^{3}$ and $y \in B^{3}$, therefore $U \subset B^{3}$.

Now suppose int $U \cap B^{3}=\emptyset$. Let $x \in \partial U \cap B^{3}$. If $x \in \operatorname{int} B^{3}$, then there is $y \in \operatorname{int} U \cap B^{3}$. It contradicts the assumption. So $x \in \partial B^{3}=S^{2}$.

For (ii): Suppose that $U$ is not a subset of $B^{3}$, then there is a point $x \in$ $U \cap\left(N-\operatorname{int} B^{3}\right)$. Let $y \in N-\operatorname{int} U$. If $y \in N-\operatorname{int} B^{3}$, there is a path $\alpha$ in $N-\operatorname{int} B^{3}$ connecting $x$ and $y$, since $N-\operatorname{int} B^{3}$ is connected. This path $\alpha$ does not meet $\partial U$, because $\partial U \subset B^{3}$. This would contradict that $x \in U$ and $y \in N-\operatorname{int} U$. Hence we must have $y \in B^{3}$, and therefore $N-\operatorname{int} U \subset B^{3}$.

Lemma 8 Suppose $H_{1}$ meets the defining conditions (1), (2) and (3) of the set $\mathcal{H}$. Then $H_{1}$ can be modified to be a 3 -submanifold $H_{*} \subset N$ such that:

(i) $\partial H_{*}$ contains no 2-sphere;

(ii) $c\left(H_{*}\right) \leq c\left(H_{1}\right)$;

(iii) $H_{*}$ still meets the the defining conditions (1) (2) (3) of $\mathcal{H}$.

Proof We suppose that $\partial H_{1}$ contains a 2-sphere component $S^{2}$, otherwise Lemma 8 is proved. Then $S^{2}$ bounds a 3 -ball $B^{3}$ in $N$ since $N$ is irreducible. We consider two cases:

Case (a) $S^{2}$ does bound a 3 -ball $B^{3}$ in $H_{1}$.

In this case $B^{3}$ is a component of $H_{1}$. By Lemma 5 , $f$ can be homotoped to be a homeomorphism outside $H_{2}=H_{1}-B^{3}$. 
Case (b) $S^{2}$ does not bound a 3 -ball $B^{3}$ in $H_{1}$.

Let $H_{1}^{\prime}$ be the component of $H_{1}$ such that $S^{2} \subset \partial H_{1}^{\prime}$. By Lemma 7 (i), either:

( $\left.\mathbf{b}^{\prime}\right) \quad H_{1}^{\prime} \subset B^{3}$, or

(b') $H_{1}^{\prime} \cap B^{3}=S^{2}$.

In case $\left(\mathbf{b}^{\prime}\right)$, let $H_{2}=H_{1}-B^{3}$. By Lemma $5 f$ can be homotoped to be a homeomorphism outside $H_{2}$. Note $H_{2} \neq \emptyset$, otherwise $M$ and $N$ are homeomorphic, which contradicts our assumption.

In case $\left(\mathbf{b}^{\prime \prime}\right)$, let $H_{2}=H_{1} \cup B^{3}$, then $\partial H_{2}$ has at least one component less than $\partial H_{1}$. Since we are enlarging $H_{1}, f$ is a homeomorphism outside $H_{2}$.

It is easy to check that in each case $(\mathrm{a}),\left(\mathrm{b}^{\prime}\right),\left(\mathrm{b}^{\prime \prime}\right)$ the components of $H_{2}$ verify the defining condition (3) of $\mathcal{H}$ and $c\left(H_{2}\right) \leq c\left(H_{1}\right)<c(H)$. Moreover $H_{2}$ is not empty because $M$ and $N$ are not homeomorphic, and $\partial H_{2} \neq \emptyset$ since $g\left(H_{2}\right) \leq g\left(H_{1}\right)<g(N)$. Hence each of the transformations (a), $\left(\mathrm{b}^{\prime}\right)$ and $\left(\mathrm{b}^{\prime \prime}\right)$ preserves properties (ii) and (iii) in the conclusion of Lemma 8 Since each one strictly reduces the number of components of $H_{1}$ or of $\partial H_{1}$, after a finite number of such transformations we reach a 3-submanifold $H_{*}$ of $N$ such that $H_{*}$ meets the properties (ii) and (iii) of Lemma 8 , and $\partial H_{*}$ contains no 2-sphere components. This proves Lemma 8 .

Lemma 9 Suppose that $H_{1}$ meets conditions (i), (ii) and (iii) in the conclusion of Lemma 8. Then $H_{1}$ can be modified to be a 3-submanifold $H_{*}$ of $N$ such that:

(a) $H_{*}$ is irreducible;

(b) $c\left(H_{*}\right) \leq c\left(H_{1}\right)$ is not increasing;

(c) $H_{*}$ still meets the the defining conditions (1), (2), (3) of $\mathcal{H}$.

In particular $H_{*}$ belongs to $\mathcal{H}$.

Proof If there is an essential 2-sphere $S^{2}$ in $H_{1}$, it must separate $N$ since $N$ is irreducible. Let $H_{1}^{\prime}$ be the component of $H_{1}$ containing $S^{2}$. The 2-sphere $S^{2}$ induces a connected sum decomposition of $H_{1}^{\prime}$ : it separates $H_{1}^{\prime}$ into two connected parts $K_{\circ}$ and $K_{\circ}^{\prime}$, such that:

$$
H_{1}^{\prime}=K \#_{S^{2}} K^{\prime}=K_{\circ} \cup_{S^{2}} K_{\circ}^{\prime},
$$

$K_{\circ} \subset H_{1}$ (resp. $K_{\circ}^{\prime} \subset H_{1}$ ) is homeomorphic to a once punctured $K$ (resp. a once punctured $\left.K^{\prime}\right)$. 
By Haken's Lemma, we have:

$$
g\left(H_{1}^{\prime}\right)=g(K)+g\left(K^{\prime}\right) .
$$

Neither $K_{\circ}$ nor $K_{\circ}^{\prime}$ is a $n$-punctured 3 -sphere, $n \geq 0$, because $\partial H_{1}$ contains no 2-sphere component, hence:

$$
g(K)<g\left(H_{1}^{\prime}\right) \quad \text { and } \quad g\left(K^{\prime}\right)<g\left(H_{1}^{\prime}\right)
$$

Since $N$ is irreducible, $S^{2}$ bounds a 3 -ball $B^{3}$ in $N$. We may assume that $\operatorname{int} K_{\circ} \cap B^{3}=\emptyset$ and int $K_{\circ}^{\prime} \cap B^{3} \neq \emptyset$. By Lemma 7 (i), we have $K_{\circ} \cap B^{3}=S^{2}$ and $K_{\circ}^{\prime} \subset B^{3}$.

Moreover $\partial H_{1}^{\prime} \cap B^{3} \neq \emptyset$, otherwise $K_{\circ}^{\prime}$ is homeomorphic to $B^{3}$, in contradiction with the assumption that $S^{2}$ is a 2 -sphere of connected sum.

Lemma $10 \partial H_{1}^{\prime}$ is not a subset of $B^{3}$.

Proof We argue by contradiction. If $\partial H_{1}^{\prime}$ is a subset of $B^{3}$, we have $N-$ $\operatorname{int} H_{1}^{\prime} \subset B^{3}$ by Lemma $\mathbf{\square}$ (ii), since $H_{1}^{\prime}$ is not a subset of $B^{3}$. Then:

$$
N=H_{1}^{\prime} \cup\left(N-\operatorname{int} H_{1}^{\prime}\right)=H_{1}^{\prime} \cup B^{3}=\left(K_{\circ} \#_{S^{2}} K_{\circ}^{\prime}\right) \cup B^{3}=K_{\circ} \cup_{S^{2}} B^{3}=K .
$$

Hence $K$ is homeomorphic to the whole $N$. If $g\left(H_{1}^{\prime}\right)<g(N)$, this contradicts the fact that $g(K)<g\left(H_{1}^{\prime}\right)<g(N)$. If $H_{1}^{\prime}$ does not carry $\pi_{1} N$ this contadicts the fact that $K \subset H_{1}^{\prime}$.

By Lemma 10, $\partial H_{1}^{\prime}$ (and therefore $\partial H_{1}$ ) has components disjoint from $B^{3}$. Therefore if we replace $H_{1}$ by $H_{2}=H_{1} \cup B^{3}$, then $\partial H_{2}$ is not empty and it has no component which is a 2-sphere. Moreover the application of Haken's Lemma above shows that $g\left(H_{2}\right)<g\left(H_{1}\right)$.

Since we are enlarging $H_{1}, f$ is a homeomorphism outside $H_{2}$, and clearly $H_{2}$ still meets the the defining condition (3) of $\mathcal{H}$. Moreover $c\left(H_{2}\right) \leq c\left(H_{1}\right)$. Hence the transformation from $H_{1}$ to $H_{2}$ preserves properties (b) and (c) in the conclusion of Lemma 9. Since it strictly reduces $g\left(H_{1}\right)$, after a finite number of such transformations we will reach a 3-submanifolds $H_{*} \subset N$ such that $H_{*}$ meets conditions (b) and (c) in the conclusion of Lemma 9, but does not contain any essential 2-sphere. This proves Lemma 9

Lemma 8 and Lemma 9 imply Lemma 6. Hence we have proved Proposition 2 under condition $(*)$. 
Proof of Proposition 2] Let $M$ and $N$ be two closed, small 3-manifolds which are not homeomorphic. Suppose there is degree one map $f: M \rightarrow N$ which is a homeomorphism outside an irreducible submanifold $H \subset N$ such that: for each component $U$ of $H$, either $g(U)<g(N)$ or $U$ does not carry $\pi_{1} N$.

Condition $(*)$ in the above proof of Proposition 2 is only used in the proof of Lemma 5 , when $H$ contains a 3-ball component $B^{3}$ and that $M-\operatorname{int} f^{-1}\left(B^{3}\right)=$ $B_{*}^{3}$ and $f^{-1}\left(B^{3}\right) \neq B_{*}^{3}$. Indeed we can now prove that this case cannot occur.

If this case happens then $\pi_{1} N=\{1\}$ and thus $m g(H)<g(N)$, since every component of $H$ carries $\pi_{1} N$. By replacing $f^{-1}\left(B^{3}\right)$ by a 3 -ball $B_{\#}^{3}$, we obtain a degree one map $\bar{f}: S^{3}=B_{*}^{3} \cup B_{\#}^{3} \rightarrow N$ defined by $\bar{f}\left|B_{*}=f\right| B_{*}$ and $\bar{f} \mid: B_{\#}^{3} \rightarrow B_{3}$ is a homeomorphism. Then $\bar{f}: S^{3} \rightarrow N$ is a map which is a homeomorphism outside a submanifold $H^{\prime}=H-B^{3}$. Clearly $m g\left(H^{\prime}\right)=$ $m g(H)<g(N)$. Furthermore condition $(*)$ now holds.

Since Proposition 2 has been proved under condition $(*)$, we have that $N$ must be homeomorphic to $S^{3}$, since $S^{3}$ does not contain any incompressible surface. It would follow that $m g(H)<0$, which is impossible.

The proof of Proposition 2, and hence of Theorem 1 is now complete.

\section{Heegaard genus of small 3-manifolds}

This section is devoted to the proof of Theorem 2

Let $M$ be a closed orientable irreducible 3-manifold. Let $F \subset M$ be a closed orientable surface (not necessary connected) which splits $M$ into finitely many compact connected 3-manifolds $U_{1}, \ldots, U_{n}$.

Let $M \backslash \mathcal{N}(F)$ be the manifold $M$ split along the surface $F$. We define the complexity of the pair $(M, F)$ as

$$
c(M, F)=\left\{\sigma(F), \pi_{0}(M \backslash \mathcal{N}(F))\right\},
$$

where $\sigma(F)$ is the sum of the squares of the genera of the components of $F$ and $\pi_{0}(M \backslash \mathcal{N}(F))$ is the number of components of $M \backslash \mathcal{N}(F)$.

Let $\mathcal{F}$ be the set of all closed surfaces $F$ such that for each component $U_{i}$ of $M \backslash F$, either $g\left(U_{i}\right)<g(M)$ or $U_{i}$ does not carry $\pi_{1} M$.

Remark 5 This condition implies that the surface $F \neq \emptyset$ for every $F \in \mathcal{F}$. 
With the hypothesis of Theorem 2, the set $\mathcal{F}$ is not empty. Let $F \in \mathcal{F}$ be a surface realizing the minimal complexity. Then the following Lemma implies Theorem 2,

Lemma 11 A surface $F \in \mathcal{F}$ realizing the minimal complexity contains no 2-sphere component and is incompressible.

Proof The arguments are analogous to those used in the proof of Propositions 2. We argue by contradiction.

Suppose that $F$ contains a 2-sphere component $S^{2}$. It bounds a 3 -ball $B^{3} \subset M$, since $M$ is irreducible. Let $U_{1}$ and $U_{2}$ be the closures of the components of $M \backslash \mathcal{N}(F)$ which contain $S^{2}$. Then by Lemma 7 (i), either:

- $U_{2} \subset B^{3}$ and $U_{1} \cap B^{3}=S^{2}$, or

- $U_{1} \subset B^{3}$ and $U_{2} \cap B^{3}=S^{2}$.

Since those two cases are symmetric, we may assume that we are in the first case. We consider the surface $F^{\prime}$ corresponding to the decomposition $\left\{U_{1}^{\prime}, \ldots, U_{k}^{\prime}\right\}$ of $M$ with $U_{1}^{\prime}=U_{1} \cup B^{3}$, after forgetting all $U_{i} \subset B^{3}$ and then re-indexing the remaining $U_{i}$ 's to be $U_{2}^{\prime}, \ldots, U_{k}^{\prime}$. This operation does not increase the Heegaard genus of any one of the components of the new decomposition. Moreover if $U_{1}$ does not carry $\pi_{1} M$, the same holds for $U_{1}^{\prime}$. Hence $F^{\prime}$ still belongs to $\mathcal{F}$. However, this operation strictly decreases the number of components of $F$, hence $c\left(F^{\prime}\right)<c(F)$, in contradiction with our choice of $F$.

Suppose that the surface $F$ is compressible. Then some essential simple closed curve $\gamma$ on $F$ bounds an embedded disk in $M$. Let $D^{\prime}$ be a such a compression disk with the minimum number of circles of intersection in $\operatorname{int} D^{\prime} \cap F$. Then a subdisk of $D^{\prime}$ bounded by an innermost circle of intersection is contained inside one of the $U_{i}$, say $U_{1}$.

Let $(D, \partial D) \subset\left(U_{1}, F \cap \partial U_{1}\right)$ be such an innermost disk. Let $U_{2}$ be adjacent to $U_{1}$ along $F$, such that $\partial D \subset \partial U_{2}$. By surgery along $D$, we get a new surface $F^{\prime}$ which gives a new decomposition $\left\{U_{1}^{\prime}, \ldots, U_{n}^{\prime}\right\}$ of $M$ as follows:

$$
U_{1}^{\prime}=U_{1} \backslash \mathcal{N}(D), \quad U_{2}^{\prime}=U_{2} \cup \mathcal{N}(D), \quad U_{i}^{\prime}=U_{i}, \text { for } i \geq 3 .
$$

Then $g\left(U_{i}^{\prime}\right) \leq g\left(U_{i}\right)$, for $i=1, \ldots, n$. Moreover if $U_{i}$ does not carry $\pi_{1} M$, the same holds for $U_{i}^{\prime}$. Hence $F^{\prime} \in \mathcal{F}$. However, $\sigma\left(F^{\prime}\right)<\sigma(F)$ since $\partial D$ is an essential circle on $F$. Therefore $c\left(F^{\prime}\right)<c(F)$ and we reach a contradiction. 
Degree one maps between small 3-manifolds and Heegaard genus

\section{Null-homotopic knot with small unknotting num- ber}

In this section we prove Theorem 3 .

Suppose $M$ is a closed, small 3-manifold and $k \subset M$ is a null-homotopic knot with $u(k)<g(M)$. Then clearly $M$ is not the 3 -sphere.

If $k$ is a non-trivial knot in a 3-ball $B^{3} \subset M$. Then the compact 3-manifold $B^{3}(k, \lambda)$ obtained by any non-trivial surgery of slope $\lambda$ on $k$ will not be a 3-ball by GL. Therefore $M(k, \lambda)$ contains an essential 2-sphere.

Hence below we assume that $k$ is not contained in a 3 -ball.

Since the knot $k \subset M$ is null-homotopic with unknotting number $u(k), k$ can be obtained from a trivial knot $k^{\prime} \subset B^{3} \subset M$ by $u(k)$ self-crossing changes. Let $D^{\prime} \subset M$ be an embedded disk bounded by $k^{\prime}$. If we let $D^{\prime}$ move following the self-crossing changes from $k^{\prime}$ to $k$, then each self-crossing change corresponds to an identification of pairs of arcs in $D^{\prime}$. Hence one obtains a singular disk $\Delta$ in $M$ with $\partial \Delta=k$ and with $u(k)$ clasp singularities. Since $\Delta$ has the homotopy type of a graph, its regular neighborhood $\mathcal{N}(\Delta)$ is a handlebody of genus $g(\mathcal{N}(\Delta))=u(k)<g(M)$.

First we prove the following lemma which is a particular case of a more general result about Dehn surgeries on null-homotopic knots, obtained in BBDM]. Since this paper is not yet available, we give here a simpler proof in this particular case.

Lemma 12 With the hypothesis above, if the slope $\alpha$ is not the meridian slope of $k$, then $M(k, \alpha)$ is not homeomorphic to $M$.

Proof Since $M$ is irreducible and $k \subset M$ is not contained in a 3-ball, $M-$ $\operatorname{int} \mathcal{N}(k)$ is irreducible and $\partial$-irreducible. Hence $1 \leq u(k)<g(M)$ and $M$ cannot be a lens space.

Let consider the set $\mathcal{W}$ of compact, connected, orientable, 3-submanifolds $W \subset$ $M$ such that:

(1) $k \subset W$ is null-homotopic in $W$;

(2) there is no 2-sphere component in $\partial W$;

(3) $g(W)<g(M)$. 
By hypothesis the set $\mathcal{W}$ is not empty since a regular neighborhood $\mathcal{N}(\Delta)$ of a singular unknotting disk for $k$ is a handlebody of genus $\geq 1$.

Claim 1 For a 3 -submanifold $W_{0} \in \mathcal{W}$ with a minimal complexity $c\left(W_{0}\right)=$ $\sigma\left(\partial W_{0}\right)$, the surface $\partial W_{0}$ is incompressible in the exterior $M-\operatorname{int} \mathcal{N}(k)$.

Proof If $\partial W_{0}$ is compressible in $M-\operatorname{int} W_{0}$, let $(D, \partial D) \hookrightarrow\left(M-\operatorname{int} W_{0}, \partial W_{0}\right)$ be a compression disk for $\partial W_{0}$. The 3-manifold $W_{1}=W_{0} \cup \mathcal{N}(D)$, obtained by adding a 2 -andle to $W_{0}$, is a compact, connected submanifold of $M$ containing $k$.

Any 2-sphere in $\partial W_{1}$ bounds a 3 -ball in $M-\operatorname{int} \mathcal{N}(k)$ since it is irreductible. Hence after gluing some 3-ball along the boundary, we may assume that $W_{1}$ contains no 2-sphere component. Moreover $k \subset W_{1}$ is null-homotopic in $W_{1}$ and $g\left(W_{1}\right) \leq g\left(W_{0}\right)<g(M)$. It follows that $W_{1} \in \mathcal{W}$. Since $c\left(W_{1}\right)<c\left(W_{0}\right)$ we get a contradiction.

If $\partial W_{0}$ is compressible in $W_{0}-\operatorname{int} \mathcal{N}(k)$, let $(D, \partial D) \hookrightarrow,\left(W_{0}-\operatorname{int} \mathcal{N}(k), \partial W_{0}\right)$ be a compression disk for $\partial W_{0}$. Let $W_{2}$ be the component of the 3-manifold $W_{0} \backslash \mathcal{N}(D)$ which contains $k$. As above, after possibly gluing some 3-ball along the boundary, we may assume that $\partial W_{2}$ contains no 2-sphere component. The knot $k \subset W_{2}$ is null-homotopic in $W_{2}$, since it is null-homotopic in $W_{0}$ and $\pi_{1} W_{2}$ is a factor of the free product decomposition of $W_{0}$ induced by the $\partial-$ compression disk $D$. Moreover by Lemma $19\left(W_{2}\right) \leq g\left(W_{0}\right)<g(M)$. It follows that $W_{2} \in \mathcal{W}$ and $c\left(W_{2}\right)<c\left(W_{0}\right)$. As above this contradicts the minimality of $c\left(W_{0}\right)$.

To finish the proof of Lemma 12 we distinguish two cases:

(a) The surface $\partial W_{0}$ is compressible in $W_{0}(k, \alpha)$ Then one can apply Scharlemann's theorem [Sch, Thm 6.1]. The fact that $k \subset W_{0}$ is null-homotopic rules out cases a) and b) of Scharlemann's theorem. Moreover by [BW, Prop.3.2] there is a degree one map $g: W_{0}(k, \alpha) \rightarrow W_{0}$, and thus there is a simple closed curve on $\partial W_{0}$ which is a compression curve both in $W_{0}(k, \alpha)$ and in $W_{0}$. Therefore case d) of Scharlemann's theorem cannot occure. The remaining case $c$ ) of Scharlemann's theorem shows that $k \subset W_{0}$ is a non-trivial cable of a knot $k_{0} \subset W_{0}$ and that the surgery slope $\alpha$ corresponds to the slope of the cabling annulus. But then the manifold $M(k, \alpha)$ is the connected sum of a non-trivial Lens space with a manifold obtained by Dehn surgery along $k_{0}$. If $M(k, \alpha)$ is homeomorphic to the small 3-manifold $M$, then $M$ and $M(k, \alpha)$ both would be homeomorphic to a Lens space, which is impossible since $1 \leq u(k)<g(M)$. 
(b) The surface $\partial W_{0}$ is incompressible in $W_{0}(k, \alpha)$ Since $\partial W_{0}$ is incompressible in $M-\mathcal{N}(k)$, it is incompressible in $M(k, \alpha)$. Therefore $M(k, \alpha)$ and $M$ cannot be homeomorphic since $M$ is a small manifold.

It follows from [BW, Prop.3.2] that there is a degree one map $f: M(k, \alpha) \rightarrow M$ which is a homeomorphism outside $\mathcal{N}(\Delta)$. Since $g(\mathcal{N}(\Delta))=u(k)<g(M)$, Theorem 3 is a consequence of Theorem 1 and Lemma 12 .

\section{References}

[BBDM] M Boileau, S Boyer, M Domergue, Y Mathieu, Killing slopes, in preparation

[BW] M Boileau, S Wang, Non-zero degree maps and surface bundles over $S^{1}$, J. Differential Geom. 43 (1996) 789-806 MathReview

[BO] F Bonahon, J-P Otal, Scindements de Heegaard des espaces lenticulaires, Ann. Sci. École Norm. Sup. (4) 16 (1983) 451-466 (1984) MathReview

[Br] W Browder, Surgery on simply-connected manifolds, Springer-Verlag, New York (1972) MathReview

[CG] A J Casson, C M Gordon, Reducing Heegaard splittings, Topology Appl. 27 (1987) 275-283 MathReview

[Ed] A L Edmonds, Deformation of maps to branched coverings in dimension two, Ann. of Math. (2) 110 (1979) 113-125 MathReview

[GL] C McA Gordon, J Luecke, Reducible manifolds and Dehn surgery. Topology 35 (1996) 385-409 MathReview

[Ha] W Haken, On homotopy 3-spheres, Illinois J. Math. 10 (1966) 159-178 MathReview

[He] J Hempel, 3-Manifolds, Princeton University Press, Princeton, N. J. (1976) MathReview

[Ja] W H Jaco, Lectures on three-manifold topology, CBMS Regional Conference Series in Mathematics 43, American Mathematical Society, Providence, RI (1980) MathReview

[La] M Lackenby, The Heegaard genus of amalgamated 3-manifolds, Geom. Dedicata 109 (2004) 139-145 MathReview

[Le] F Lei, Complete systems of surfaces in 3-manifolds, Math. Proc. Cambridge Philos. Soc. 122 (1997) 185-191 MathReview

[RW] Y W Rong, S C Wang, The preimages of submanifolds, Math. Proc. Cambridge Philos. Soc. 112 (1992) 271-279 MathReview 
[Sch] M Scharlemann, Producing reducible 3-manifolds by surgery on a knot, Topology 29 (1990) 481-500 MathReview

[Wa] F Waldhausen, On mappings of handlebodies and of Heegaard splittings, from: "Topology of Manifolds (Proc. Inst. Univ. of Georgia, Athens, Ga. 1969)", Markham, Chicago, Ill. (1970) 205-211 MathReview

Laboratoire Émile Picard, CNRS UMR 5580, Université Paul Sabatier 118 Route de Narbonne, F-31062 TOULOUSE Cedex 4, France

and

LAMA Department of Mathematics, Peking University

Beijing 100871, China

Email: boileau@picard.ups-tlse.fr, wangsc@math.pku.edu.cn

Received: 20 July 2005 